\title{
Enhancement of ketoconazole dissolution rate by the liquisolid technique
}

\author{
MIR-ALI MOLAEI ${ }^{1,5}$ \\ KARIM OSOULI-BOSTANABAD ${ }^{2,3}$ \\ KHOSRO ADIBKIA ${ }^{2,4}$ \\ JAVAD SHOKRI ${ }^{4}$ \\ SOLMAZ ASNAASHARI ${ }^{5}$ \\ YOUSEF JAVADZADEH ${ }^{4,5 *}$ \\ ${ }^{1}$ Drug Applied Research Center, Tabriz \\ University of Medical Sciences, Tabriz \\ 51664, Iran \\ ${ }^{2}$ Center for Pharmaceutical \\ Nanotechnology, Tabriz University of \\ Medical Sciences, Tabriz 51664, Iran \\ ${ }^{3}$ Students Research Committee, Tabriz \\ University of Medical Sciences, Tabriz \\ Iran \\ ${ }^{4}$ Faculty of Pharmacy, Tabriz \\ University of Medical Sciences, Tabriz \\ 51664, Iran \\ ${ }^{5}$ Biotechnology Research Center, Tabriz \\ University of Medical Sciences, Tabriz \\ 51664, Iran
}

Accepted March 15, 2018

Published online April 27, 2018

\begin{abstract}
The study was conducted to enhance the dissolution rate of ketoconazole (KCZ) (a poorly water-soluble drug) using the liquisolid technique. Microcrystalline cellulose, colloidal silica, PEG400 and polyvinyl pyrrolidone (PVP) were employed as a carrier, coating substance, nonvolatile solvent and additive in the KCZ liquisolid compact formulation, respectively. The drug-to-PEG400 and carrier-to-coating ratio variations, PVP concentration and aging effects on the in vitro release behavior were assessed. Differential scanning calorimetry (DSC) and X-ray powder diffraction (XRD) data revealed no alterations in the crystalline form of the drug and the KCZ-excipient interactions within the process. The load factor and the drug release rate were significantly enhanced compared to directly compressed tablets in the presence of the additive. Increasing the PEG400to-drug ratio in liquid medications enhanced the dissolution rate remarkably. The dissolution profile and hardness of liquisolid compacts were not significantly altered by keeping the tablets at $40{ }^{\circ} \mathrm{C}$ and relative humidity of $75 \%$ for 6 months. With the proposed modification of the liquisolid process, it is possible to obtain flowable, compactible liquisolid powders of high-dose poorly-water soluble drugs with an enhanced dissolution rate.
\end{abstract}

Keywords: ketoconazole, liquisolid, dissolution rate, Avicel, polymorphic changes

Dissolution of active elements of a solid dosage form with low aqueous solubility is an important issue that should be achieved before its absorption via the gastrointestinal (GI) tract (1). For pharmaceutical scientists, the low dissolution features of water-insoluble drugs are a major challenge $(2,3)$. The dissolution rate of a drug with low aqueous solubility at its absorption site is the main controlling parameter that affects the absorption rate of the drug prepared as an orally delivered solid dosage form, i.e. release rate is the rateverifying step in drug absorption (4). There are numerous techniques for enhancing the dissolution rate of these drugs, including (a) size reduction to increase the surface area ( 3 , 5); (b) solubilization by surfactants $(3,5,6)$; (c) creation of water-soluble complexes $(3,7)$; (d)

\footnotetext{
*Correspondence; e-mail: javadzadehy@yahoo.com; javadzadehy@tbzmed.ac.ir
} 
use of pro-drug and drug derivatives such as strong electrolyte salt forms that generally have a superior dissolution rate (8); and (e) manipulation of the solid state of drugs in order to obtain better drug dissolution by reducing the crystallinity of drug material through construction of solid solutions (9). The most common method is micronization to increase the drug surface area but, in practice, the outcome of micronization is usually unsatisfactory, particularly when the drugs are formulated as capsules or tablets (10). As a consequence of hydrophobicity, micronized drugs have a tendency to agglomerate; hence, their available surface area will decrease. Several studies have shown that the most reliable technique is the liquisolid method for enhancing the release rate of drugs with poor solubility in aqueous media, which has been effectively used for such drugs (11-13). However, preparing high doses of drugs into liquisolid compacts is one of the limitations of this method. In this case, in order to obtain satisfactory flowability and compatibility of liquisolid powders, the carrier and coating substances should be added at high levels to increase the weight of each tablet and make it difficult to swallow $(2,3,14-16)$. As reported in our previous study, by adding some compounds like polyvinyl pyrrolidone (PVP) to liquid medications (microsystems), it is possible to manufacture suitable liquisolid tablets containing high drug concentrations. By doing so, a lower quantity of carrier is needed to obtain dry powders with free flowability and acceptable compatibility, as shown by carbamazepine liquisolid tablets with a remarkably increased dissolution rate (14). The reasons for dissolution rate enhancement of liquisolid formulations in the presence of PVP are mainly attributed to inhibition of crystal growth (i.e., PVP inhibits drug precipitation from a supersaturated liquid medication) as well as to the increase of the exposed drug surface area in the dissolution medium due to adsorption on the carrier (14).

Ketoconazole $(\mathrm{KCZ})$ is one of the poorly soluble drugs with high permeability in GI (class II) and its release rate in the GI tract controls its oral absorption rate $(17,18)$. Consequently, in addition to permeability, the dissolution rate and solubility of the drug are crucial for its oral bioavailability (19-21). GI injuries may also increase due to this unfavorable characteristic that increases the duration of the drug's contact with the GI mucosa; therefore, enhancement of the dissolution rate of poorly soluble drugs by liquisolid compacts appears to be a good choice. Some methods have been used to speed up the dissolution of KCZ, including the use of solid dispersions $(22,23)$ and the formation of soluble complexes with additives such as cyclodextrin $(24,25)$, with varying degrees of success.

In this study, the capability of the liquisolid technique was examined by preparing liquisolid systems of $\mathrm{KCZ}$ in order to increase its dissolution rate as a water-insoluble drug. To this end, $\mathrm{KCZ}$ was formulated in liquisolid tablets with various drug-to-solvent ratios at the same concentrations of powder excipients and PVP in their liquid medications. The in vitro drug release study of liquisolid systems was conducted by comparing the dissolution behavior of directly compressed tablets (DC) using a USP apparatus II-paddle stirrer in SGF dissolution media $(\mathrm{pH}=1.2)$.

\section{EXPERIMENTAL}

\section{Materials}

Ketoconazole was provided by Sobhan Co. (Rasht, Iran). Cellulose with a coarse granular microcrystalline structure (Avicel PH101), amorphous silicon dioxide (silica) in nano- 
meter size and sodium starch glycolate were supplied by Mingtai Chemical (Taiwan) and Yung Zip Chemical (Taiwan), respectively. Polysorbate 80 (Tween 80), propylene glycol (PG), polyethylene glycol 400 (PEG), PEG 200, glycerin, industrial grade corn starch, monohydrate lactose, sorbitol and sodium chloride were purchased from Merck (Germany). Polyvinyl pyrrolidone K25 (PVP) was obtained from BASF (Germany) and hydroxypropyl methylcellulose K4M (HPMC) was purchased from Colorcon (England). All the other chemical substances were of analytical grade.

\section{Preparation of conventional and liquisolid tablets}

KCZ regular tablets (directly compressed tablets (DC) or physical mixture (PM)) were formulated by blending KCZ with microcrystalline cellulose and silica. The blend was agitated for $10 \mathrm{~min}$ with sodium starch glycolate (SSG) (as a disintegrating agent). Then, a die with a $10-\mathrm{mm}$ punch was used to compress the blend using a manual tableting machine (Riken, Japan) and applying adequate compression load to fabricate compacts of 7-8 SC hardness (Strong Cobb). Each tablet contained $100 \mathrm{mg}$ of KCZ, $400 \mathrm{mg}$ of cellulose (as a carrier), $20 \mathrm{mg}$ of silica in nanometer range (as a coating substance) and $25 \mathrm{mg}$ of SSG.

Different liquisolid compacts (Table I) were also prepared. KCZ was dispersed in PEG 400 (liquid medication was prepared using PEG 400 as solvent). PVP was then dissolved in PEG 400 containing ketoconazole. After that, a binary blend of the carrier and coating substances was added to the resultant liquid medication under continuous agitation in a mortar and finally mixed with $5 \%(\mathrm{~m} / \mathrm{m})$ of SSG as disintegrant. The final mixture was compacted using the manual tableting machine to obtain a compact hardness of 7-8 SC. Key formulation features of the prepared KCZ liquisolid formulations are given in Table I.

\section{Drug content evaluation test}

Ten tablets were weighed and ground into a fine powder. The amount of powder equivalent to $200 \mathrm{mg}$ of KCZ was weighed accurately into a $100 \mathrm{~mL}$ calibrated flask, $70 \mathrm{~mL}$

Table I. Key formulation characteristics of the prepared ketoconazole liquisolid compacts

\begin{tabular}{|c|c|c|c|}
\hline Components $\quad$ Formulation & F1 & F2 & F3 \\
\hline Drug (g) & 10 & 13.33 & 16.66 \\
\hline PEG 400 (mL) & 20 & 16.66 & 13.33 \\
\hline PVP (g) & 3.33 & 3.33 & 3.33 \\
\hline Avicel (g) & 77.52 & 77.52 & 77.52 \\
\hline Silica (g) & 5.2 & 5.2 & 5.2 \\
\hline Starch (g) & 5.8 & 5.8 & 5.8 \\
\hline $\begin{array}{l}\text { Weight of each compacted } \\
\text { liquisolid tablet (g) }\end{array}$ & 1.218 & 0.914 & 0.731 \\
\hline
\end{tabular}


of ethanol was added, and the flask was shaken for about $12 \mathrm{~h}$. Then, the volume was made up and filtered using Whatmann No 42 filter paper. The filtrate was diluted appropriately and analyzed by spectrophotometry.

\section{Solubility studies}

PEG 200, PEG 400, glycerin, polysorbate 80 and PG, as five different nonvolatile solvents, were employed and their solubility was studied to determine the proper dissolution or suspension of the nonvolatile solvent of $\mathrm{KCZ}$ in liquid medications. In this regard, saturated drug-solvent solutions were made by putting an excess drug into the vessel and agitating it on a shaker (Velp, Italy) under constant vibration at $25^{\circ} \mathrm{C}$ for $48 \mathrm{~h}$. The solutions were filtered through a Millipore filter with a pore diameter of $0.45 \mu \mathrm{m}$, then diluted and evaluated at a wavelength of $284.4 \mathrm{~nm}$ with a UV spectrophotometer (Shimadzu, Kyoto, Japan). The solubility of KCZ was obtained from the mean values of three measurements for each sample (Table III).

\section{Load factor calculation}

To calculate the load factor $\left(L_{\mathrm{f}}\right)$, several concentrations $(\mathrm{m} / \mathrm{m})$ of polymer (PVP) were dissolved in PEG 400 (KCZ exhibited maximum solubility in PEG 400) (Table III). The system (liquid medication without KCZ) was incorporated into $30 \mathrm{~g}$ of the Avicel-silica powder blend as a carrier substance and mixed for $10 \mathrm{~min}$. Flowability of $100 \mathrm{~cm}^{3}$ of liquisolid systems was measured using a flowmeter (Erweka, Germany). The flow rate above $10 \mathrm{~cm}^{3} / \mathrm{s}$ was considered a suitable flow rate for tableting in this study. Load factor amounts were calculated using the formula $L_{\mathrm{f}}=W / Q$, where $W$ and $Q$ show the liquid medication and carrier material concentrations, respectively. The resultant $L_{\mathrm{f}}$ was used to obtain the amount of coating and carrier substances in each formulation. The $L_{\mathrm{f}}$ obtained in this study was 0.44 .

\section{Spectrophotometric analysis}

UV/visible spectrophotometry (Shimadzu-120, Kyoto, Japan) was used for the analysis of all the KCZ samples at $223.4 \mathrm{~nm}$. Serially diluted aqueous stock solutions of the drug in simulated gastric fluids (SGF), $\mathrm{pH}=1.2$, were used to assemble standard curves and attain

Table II. Results of the spectrophotometric analysis of ketoconazole

\begin{tabular}{cc}
\hline Abs. & Conc. $\left(\mu \mathrm{g} \mathrm{mL}^{-1}\right)$ \\
\hline 0.064 & 1.56 \\
0.111 & 3.12 \\
0.267 & 6.25 \\
0.613 & 12.50 \\
1.970 & 50.00 \\
\hline
\end{tabular}


concentrations in the range of $1.5-50 \mu \mathrm{g} \mathrm{mL}^{-1}$. Data of each curve were acquired using the mean values of three measurements. Results of the spectrophotometric analysis are summarized in Table II.

\section{Powder X-ray diffraction analysis}

X-ray measurements of ground powders of the pure drug, PM/DC, liquisolid formulations and excipients were made with the aid of X-ray diffraction (Siemens, Model D5000 X-ray Diffractometer, Germany) on a quartz plate (with a low background) with a step size of $0.02^{\circ}$, at a scanning rate of $0.6^{\circ} \mathrm{min}$, using $\mathrm{Cu} K \alpha$ radiation $\left(k=1.5405 \mathrm{~A}^{\circ}\right)$ from 5 to $70^{\circ}$ working at $40 \mathrm{kV}$ and $30 \mathrm{~mA}$.

\section{Differential scanning calorimetry (DSC)}

A differential scanning calorimeter (DSC 60, Shimadzu, Japan) was used to examine the thermal attitude and thermograms of the samples. To this aim, the test samples were meticulously weighed (5 mg), then placed in sealed aluminum pans and a Shimadzu crimper was used to crimp the lids. A scanning rate of $20^{\circ} \mathrm{C}$ per minute was used to evaluate the thermal attitude of the samples in the range of $30-200{ }^{\circ} \mathrm{C}$. Indium powder was employed as the standard.

\section{In vitro drug release}

A USP apparatus II-paddle stirrer (Erweka, DPT6R, Germany) was used to study release profiles of the pure drug, PM/DC and the liquisolid compressed tablets. The samples, all containing $100 \mathrm{mg}$ of ketoconazole, were placed in a vessels containing $900 \mathrm{~mL}$ of simulated gastric fluid $(\mathrm{pH}=1.2)$ under rotational agitation of $100 \pm 2 \mathrm{rpm}$ at $37 \pm 0.1{ }^{\circ} \mathrm{C}$. At specified intervals $(5,10,15,20,30,45,60$ and $90 \mathrm{~min}), 5 \mathrm{~mL}$ of the prepared solutions was taken and filtered through a Millipore membrane with a pore diameter of $0.45 \mu \mathrm{m}$. After that, an equal amount of fresh dissolution fluid was added to the solution so as to retain a constant volume. To evaluate and record the cumulative drug release plot, UV spectrophotometry (Shimadzu, Kyoto, Japan) was conducted at a wavelength of $284.4 \mathrm{~nm}$. In each of the diagrams, data were attained from the mean values of three measurements.

\section{Optimized formulation stability analysis}

Optimized liquisolid compressed tablets were kept for six months at $40{ }^{\circ} \mathrm{C}$ under relative humidity of $75 \%$ in a sealed aluminum package to analyze the stability of the drug and liquisolid formulation according to the ICH standards. Drug hardness, content and its dissolution behavior were evaluated at specific intervals.

\section{Statistical analysis}

One-way ANOVA and Tukey's multiple comparison tests were used for comparison of data between the samples. Statistical significance was set at $p<0.05$. All the data were reported as mean \pm standard deviations attained from three experimented samples. 


\section{RESULTS AND DISCUSSION}

\section{Evaluation of the solid state properties of samples (XRD analysis)}

Drug precipitation might occur after its solution is adsorbed to the adsorbent. In other words, precipitation possibility depends on the drug dissolution and saturation degree into the solvent and solution or components' interaction. It has been demonstrated that the dissolution behavior and bioavailability of the drug are crucial aspects that may be influenced because of polymorphic changes (26). Therefore, the study of KCZ polymorphic changes in liquisolid formulations is important. Fig. 1 illustrates the XRD patterns of the pure drug, pure excipients, PM/DC (ketoconazole, cellulose, PVP, silica and SSG) and the liquisolid formulation. Distinct diffraction peaks at $2 \theta$ angles of 15.36, 19.56, 25, 27.47 and 14, 22, 34 are the characteristic peaks of KCZ and cellulose (Avicel), respectively, which reflect their crystalline nature $(27,28)$. The PVP, silica and starch did not show any distinctive peaks in XRD patterns, illustrating their amorphous nature. The pattern of the PM/DC and liquisolid systems had distinct diffraction peaks of the drug and one of cellulose peaks; however, intensity reduction of the peaks might be due to the dilution effect of the polymer and solubilization of the drug in liquisolid formulations.

\section{In vitro drug solubility studies}

Solubility of KCZ in various nonvolatile solvents (PG, PEG 400, PEG 200, glycerin, SGF and Tween 80 ) was evaluated to determine the drug solubility patterns in different media (Table III).

As indicated in Table III, the solubility order was PEG $400>$ PEG $200>$ PG $>$ glycerin $>$ Tween $80>$ SGF, revealing that PEG 400 had the highest solubility $\left(42.21 \pm 2.07 \mathrm{mg} \mathrm{mL}^{-1}\right)$ of the drug, with its large fraction being in molecular state. The drug dissolution rate can be directly related to its molar fraction in the liquid medication; therefore, PEG 400 was

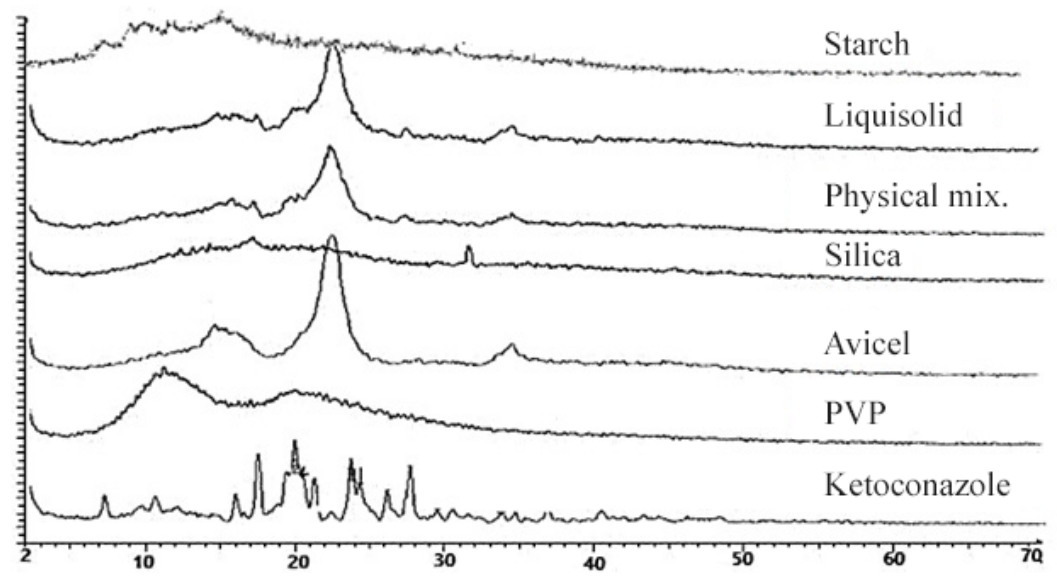

Fig. 1. X-ray diffraction patterns of pure ketoconazole, starch, PVP, Avicel, silica, physical mixture and liquisolid formulation. 
Table III. Solubility of ketoconazole in various solvents

\begin{tabular}{|c|c|c|}
\hline Solvents $\quad$ Solubility & $\mathrm{mmol} \mathrm{mL} L^{-1}$ & $\mathrm{mg} \mathrm{mL}^{-1}$ \\
\hline PG & 0.053 & 27.952 \\
\hline PEG 400 & 0.079 & 42.212 \\
\hline PEG 200 & 0.070 & 37.390 \\
\hline Glycerin & 0.004 & 2.365 \\
\hline Tween 80 & 0.002 & 1.293 \\
\hline SGF & $1.75 \times 10^{-5}$ & 0.0093 \\
\hline
\end{tabular}

selected as the best nonvolatile solvent and used in liquisolid formulations. In fact, based on the Noyes and Whitney equation, the drug dissolution rate could be increased by increasing the drug surface area; therefore, according to the results (Table III), PEG 400 contains the largest fraction of the drug in the molecular form and this would enhance the drug dissolution rate due to the increased surface area (29). In addition, the load factor and dissolution efficiency data presented in Table I confirm these results; in other words, as mentioned in our previous study (14), addition of PVP as an additive to liquisolid microsystems (liquisolid formulations) enhances the load factor and improves the flowability and compatibility of liquisolid compressed tablets in comparison with DC tablets, resulting in an improvement of the dissolution rate.

\section{DSC evaluation}

In order to recognize the physical properties of the samples and detect any probable interaction between the drug and excipients, the pure drug, excipients, PM/DC and liquisolid formulation were subjected to DSC analysis (Fig. 2). The drug revealed a distinct

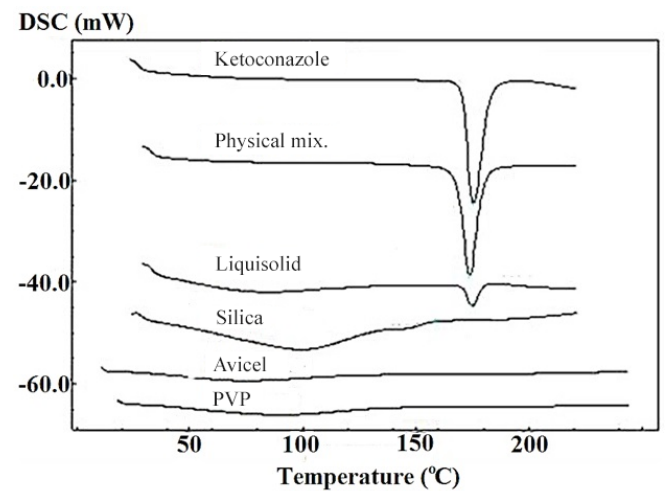

Fig. 2. DSC thermograms of pure ketoconazole, PVP, Avicel, silica, physical mixture and liquisolid formulation. 
endothermic peak at $148.6 \pm 0.2{ }^{\circ} \mathrm{C}$ corresponding to its melting point; in addition, the PM and liquisolid formulation indicated a similar peak in this region. Therefore, based on the XRD and DSC results, it can be concluded that there were no crystallinity changes in the drug or interactions between the excipients and drug throughout the formulation procedures.

However, reduced intensity of the endothermic peak of the drug in the PM and liquisolid formulation could be linked to the polymer dilution effect and/or solubilization of the drug in the melted polymer. These findings were fully consistent with XRD patterns.

\section{In vitro drug solubility and release studies}

In vitro dissolution tests were carried out to analyze the release behavior of $\mathrm{KCZ}$ from the DC and liquisolid formulations (Fig. 3). The liquisolid compressed samples showed faster release patterns compared to the DC samples at the same $\mathrm{pH}$. It was found that liquisolid formulations (F3, F2 and F1) released at least $40-53 \%$ of the drug within 30 min, while DC samples had a drug release rate of about $17 \%$ during this period. In addition, nearly $47-60 \%$ of the drug was released from liquisolid formulations in $90 \mathrm{~min}$, while about $21 \%$ was released from DC tablets within the same period of time (Fig. 3). The molecular dispersion of the drug in PEG 400 was one of the main parameters that were most likely to affect the release rate of the drug from liquisolid formulations. In other words, the molecularly dispersed drug can dissolve immediately after its disintegration in dissolution media and enhance the dissolution rate. The other reason for the enhanced release rate of liquisolid formulations compressed with DC samples could be attributed to their increased surface area, i.e., as mentioned previously, the increased dissolution rate could be strongly connected to the fact that KCZ already exists in liquid medications (PEG 400 solution) while powder particles of the liquisolid formulation (cellulose and silica) are carrying it; consequently, its accelerated release was mainly due to surface availability and wettability enhancement in the dissolution environment $(14-16,30)$.

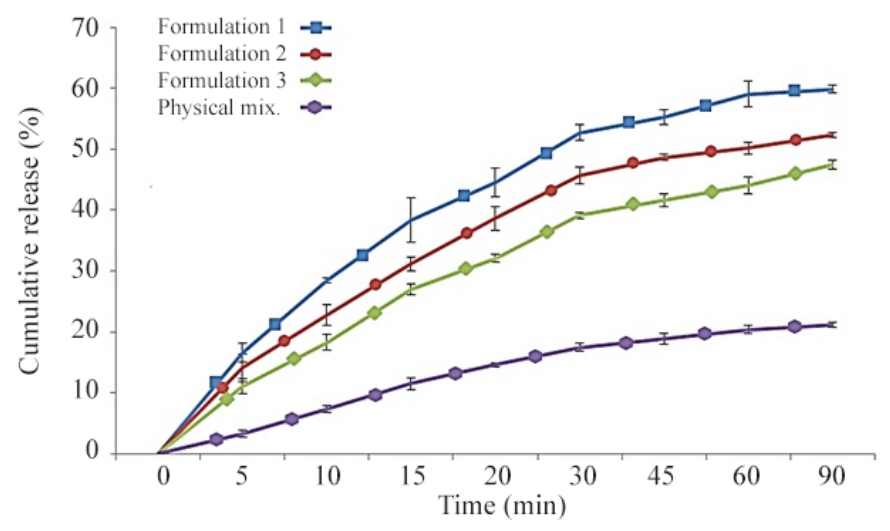

Fig. 3. Release profiles of ketoconazole in different formulations measured in simulated gastric fluid (mean $\pm \mathrm{SD}, n=3$ ). 


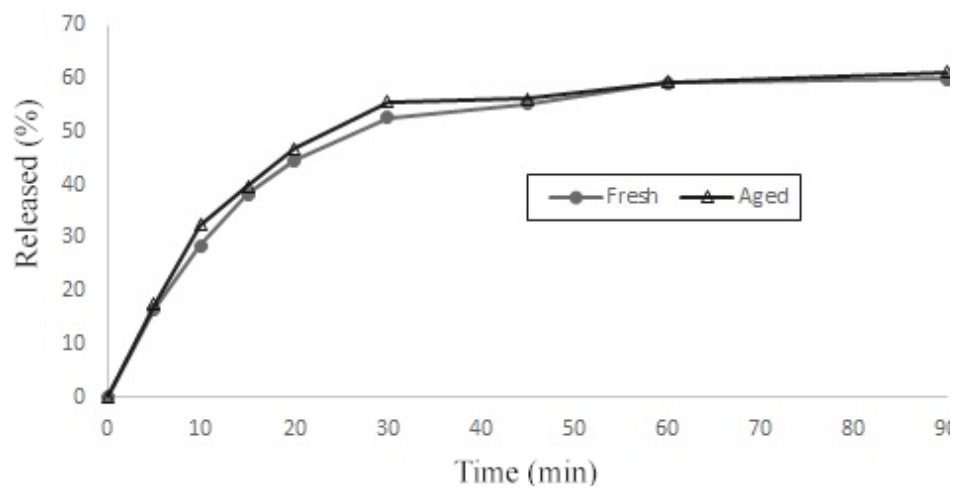

Fig. 4. Effect of aging on dissolution profiles of ketoconazole liquisolid tablets (mean $\pm \mathrm{SD}, n=3$ ).

Another reason that could explain the enhanced release rate of liquisolid compacts was their formulation and incorporation of PVP as an additive. PVP might inhibit the drug crystal growth (i.e., its precipitation from the supersaturated solution) (14) and increase the drug exposed surface area in the dissolution environment due to its adsorption on the carrier. Furthermore, a larger amount of the molecularly dissolved drug (in PEG 400) could be absorbed on the silica surface (because of its large surface area) (14-16), increasing the exposed drugs in the dissolution medium. Consequently, it would enhance the dissolution rate of liquisolid formulations. Furthermore, the graphs presented in Fig. 3 imply that an increase in drug concentration or a decrease in PEG 400 concentration in liquisolid formulations would result in a decrease in the dissolution rate of liquisolid compacts. This might be explained by changes in the solubility or the molecularly dispersed form of the drug in liquisolid systems. Moreover, it can be concluded from the DSC and XRD data that the dissolution rate enhancement of KCZ liquisolid tablets was not due to the formation of complexes between $\mathrm{KCZ}$ and excipients or alterations in $\mathrm{KCZ}$ crystallinity.

\section{Stability evaluation of liquisolid systems}

Stability evaluations were conducted on optimized formulations to reveal the effect of aging on the hardness and dissolution behavior of KCZ liquisolid compressed tablets. The results of dissolution rate and hardness measurements showed no significant variations between the hardness of aged $(8.5 \pm 1.1 \mathrm{SC})$ and fresh $(8.9 \pm 1 \mathrm{SC})$ liquisolid compacts $(p>0.05)$. Therefore, the aging process did not affect the hardness of liquisolid compressed tablets. Dissolution graphs of the aged and fresh liquisolid compacts showed a lower release rate for aged liquisolid tablets compared to the fresh ones (Fig. 4). However, considering the similarity factor of the two dissolution profiles, it can be concluded that acceptable similarity exists between the two profiles, indicating that the dissolution behavior of $\mathrm{KCZ}$ optimized formulations was not affected by the aging process. As a result, one can suppose that liquisolid tablets of KCZ will preserve their initial hardness and enhanced release behavior after 6 months. 


\section{CONCLUSIONS}

By adding PVP to a liquid medication, it is possible to load a higher amount of drug into liquisolid tablets. This is valuable for the preparation of liquisolid tablets using high dose drugs. Liquisolid tablets prepared using PVP as an additive into the liquid medication resulted in better dissolution profiles compared to DC compacts and other formulations Furthermore, no crystalline structure transformation or interaction was detected during the procedure. Finally, aging had no influence on the hardness and release profile of liquisolid compacts.

Acknowledgments. - The authors thank the Drug Applied Research Center of Tabriz University of Medical Sciences for financial support to this study.

\section{REFERENCES}

1. M. Wesoły, K. Cal, P. Ciosek and W. Wróblewski, Influence of dissolution-modifying excipients in various pharmaceutical formulations on electronic tongue results, Talanta 162 (2017) 203-209; https://doi.org/10.1016/j.talanta.2016.10.018

2. A. Nokhodchi, C. M. Hentzschel and C. S. Leopold, Drug release from liquisolid systems: speed it up, slow it down, Expert Opin. Drug Deliv. 8 (2011) 191-205; https://doi.org/10.1517/17425247.2011. 548801

3. L. Azharshekoufeh, J. Shokri, M. B. Jalali and Y. Javadzadeh, Liquigroud technique: a new concept for enhancing dissolution rate of glibenclamide by combination of liquisolid and co-grinding technologies, BioImpacts 7 (2017) 5-12; https://doi.org/10.15171/bi.2017.02

4. A. B. Karmarkar, I. D. Gonjar and A. H. Hosmani, Liquisolid technology for dissolution rate enhancement or sustained release, Expert Opin. Drug Deliv. 7 (2010) 1227-1234; https://doi.org/10.151 7/17425247.2010.511173

5. I. Theochari, M. Goulielmaki, D. Danino, V. Papadimitriou, A. Pintzas and A. Xenakis, Drug nanocarriers for cancer chemotherapy based on microemulsions: the case of Vemurafenib analog PLX4720, Colloids Surf. B Biointerfaces 154 (2017) 350-356; https://doi.org/10.1016/j.colsurfb.2017.03.032

6. M. Colombo, S. Staufenbiel, E. Rühl and R. Bodmeier, In situ determination of the saturation solubility of nanocrystals of poorly soluble drugs for dermal application, Int. J. Pharm. 521 (2017) 156-166; https://doi.org/10.1016/j.ijpharm.2017.02.030

7. K. Sigfridsson, P. Skantze, U. Skantze, L. Svensson, L. Löfgren, P. Nordell, E. Michaëlsson, B. Smedsrød, B. Fuglesteg and K. Elvevold, Nanocrystal formulations of a poorly soluble drug. 2. Evaluation of nanocrystal liver uptake and distribution after intravenous administration to mice, Int. J. Pharm. 524 (2017) 248-256; https://doi.org/10.1016/j.ijpharm.2017.03.062

8. R. Ghanavati, A. Taheri and A. Homayouni, Anomalous dissolution behavior of celecoxib in PVP/ Isomalt solid dispersions prepared using spray drier, Mater. Sci. Eng. C 72 (2017) 501-511; https:// doi.org/10.1016/j.msec.2016.11.042

9. K. I. Shingel, M. Selyanin, M. C. Filion and F. Polyak, Solid dispersions of drugs in hyaluronan matrix: The role of the biopolymer in modulating drug activity in vivo, J. Drug Deliv. Sci. Technol. 39 (2017) 140-146; https://doi.org/10.1016/j.jddst.2017.03.018

10. P. Veres, M. Kéri, I. Bányai, I. Lázár, I. Fábián, C. Domingo and J. Kalmár, Mechanism of drug release from silica-gelatin aerogel. Relationship between matrix structure and release kinetics, Colloids Surf. B Biointerfaces 152 (2017) 229-237; https://doi.org/10.1016/j.colsurfb.2017.01.019 
11. M. Lu, H. Xing, T. Yang, J. Yu, Z. Yang, Y. Sun and P. Ding, Dissolution enhancement of tadalafil by liquisolid technique, Pharm. Dev. Technol. 22 (2017) 77-89; https://doi.org/10.1080/10837450.2016 .1189563

12. K. T. Savjani, A. K. Gajjar, J and K. Savjani, Drug solubility: Importance and enhancement techniques, ISRN Pharm. 2012 (2012) 1-10; https://doi.org/10.5402/2012/195727

13. T. L. Rogers, K. A. Overhoff, P. Shah, P. Santiago, M. J. Yacaman, K. P. Johnston and R. O. Williams, Micronized powders of a poorly water soluble drug produced by a spray-freezing into liquidemulsion process, Eur. J. Pharm. Biopharm. 55 (2003) 161-172; https://doi.org/10.1016/S09396411(02)00193-5

14. Y. Javadzadeh, B. Jafari-Navimipour and A. Nokhodchi, Liquisolid technique for dissolution rate enhancement of a high dose water-insoluble drug (carbamazepine), Int. J. Pharm. 341 (2007) 26-34; https://doi.org/10.1016/j.ijpharm.2007.03.034

15. Y. Javadzadeh, M. R. Siahi, S. Asnaashari and A. Nokhodchi, Liquisolid technique as a tool for the enhancement of poorly water-soluble drugs and evaluation of their physicochemical properties, Acta Pharm. 57 (2007) 99-109; https://doi.org/10.2478/v10007-007-0008-6

16. Y. Javadzadeh, M. Siahi-Shadbad, M. Barzegar-Jalali and A. Nokhodchi, Enhancement of dissolution rate of piroxicam using liquisolid compacts, Il Farmaco 60 (2005) 361-365; https://doi. org/10.1016/j.farmac.2004.09.005

17. V. De Campos, C. Cerqueira-Coutinho, F. Capella, B. Soares, C. Holandino and C. Mansur, Development and in vitro assessment of nanoemulsion for delivery of ketoconazole against Candida albicans, J. Nanosci. Nanotechnol. 17 (2017) 4623-4630; https://doi.org/10.1166/jnn.2017.13445

18. P. Deng, F. Teng, F. Zhou, Z. Song, N. Meng and R. Feng, Methoxy poly (ethylene glycol)-b-poly ( $\delta$-valerolactone) copolymeric micelles for improved skin delivery of ketoconazole, J. Biomater. Sci. Polym. Ed. 28 (2017) 63-78; https://doi.org/10.1080/09205063.2016.1244371

19. G. A. Abdelbary, M. M. Amin and M. Y. Zakaria, Ocular ketoconazole-loaded proniosomal gels: formulation, ex vivo corneal permeation and in vivo studies, Drug Deliv. 24 (2017) 309-319; https:// doi.org/10.1080/10717544.2016.1247928

20. A. M. Stewart, M. E. Grass, D. M. Mudie, M. M. Morgen, D. T. Friesen and D. T. Vodak, Development of a biorelevant, material-sparing membrane flux test for rapid screening of bioavailabilityenhancing drug product formulations, Mol. Pharm. 14 (2017) 2032-2046; https://doi.org/10.1021/acs. molpharmaceut.7b00121

21. J. Bassi da Silva, S. B. d. S. Ferreira, O. de Freitas and M. L. Bruschi, A critical review about methodologies for the analysis of mucoadhesive properties of drug delivery systems, Drug Dev. Ind. Pharm. 43 (2017) 1053-1070; https://doi.org/10.1080/03639045.2017.1294600

22. A. Ruff, T. Fiolka and E. S. Kostewicz, Prediction of Ketoconazole absorption using an updated in vitro transfer model coupled to physiologically based pharmacokinetic modelling, Eur. J. Pharm. Sci. 100 (2017) 42-55; https://doi.org/10.1016/j.ejps.2016.12.017

23. Y. Tsume, K. Matsui, A. L. Searls, S. Takeuchi, G. E. Amidon, D. Sun and G. L. Amidon, The impact of supersaturation level for oral absorption of BCS class IIb drugs, dipyridamole and ketoconazole, using in vivo predictive dissolution system: Gastrointestinal Simulator (GIS), Eur. J. Pharm. Sci. 102 (2017) 126-139; https://doi.org/10.1016/j.ejps.2017.02.042

24. T. Loftsson and E. Stefánsson, Cyclodextrins and topical drug delivery to the anterior and posterior segments of the eye, Int. J. Pharm. 531 (2017) 413-423; https://doi.org/10.1016/j.ijpharm.2017.04.010

25. G. Balata, M. Mahdi and R. A. Bakera, Improvement of solubility and dissolution properties of ketoconazole by solid dispersions and inclusion complexes, Indian J. Pharm. Sci. 5 (2011) 517-526; https://doi.org/10.4103/0250-474X.98995

26. K. Inaba, T. Wakuda and K. Uekama, Prostaglandins and their cyclodextrin complexes, J. Incl. Phenom. Macrocycl. Chem. 2 (1984) 467-474; https://doi.org/10.1007/BF00662213 
27. P. Kanaujia, G. Lau, W. K. Ng, E. Widjaja, M. Schreyer, A. Hanefeld, M. Fischbach, C. Saal, M. Maio and R. B. Tan, Investigating the effect of moisture protection on solid-state stability and dissolution of fenofibrate and ketoconazole solid dispersions using PXRD, HSDSC and Raman microscopy, Drug Dev. Ind. Pharm. 37 (2011) 1026-1035; https://doi.org/10.3109/03639045.2011.558091

28. A. Gryczke, S. Schminke, M. Maniruzzaman, J. Beck and D. Douroumis, Development and evaluation of orally disintegrating tablets (ODTs) containing Ibuprofen granules prepared by hot melt extrusion, Colloids Surf. B Biointerfaces. 86 (2011) 275-284; https://doi.org/10.1016/j.colsurfb.2011.04.007

29. M. Dhanaraju, K. S. Kumaran, T. Baskaran and M. S. R. Moorthy, Enhancement of bioavailability of griseofulvin by its complexation with $\beta$-cyclodextrin, Drug Dev. Ind. Pharm. 24 (1998) 583-587; https://doi.org/10.3109/03639049809085663

30. Y. Javadzadeh, M. R. Siahi, S. Asnaashari and A. Nokhodchi, An investigation of physicochemical properties of piroxicam liquisolid compacts, Pharm. Dev. Technol. 12 (2007) 337-343; https://doi. org/10.1080/10837450701247574 